\title{
THEORETICAL APPROACHES TO STUDY THE VIBRATIONAL PREDISSOCIATION OF VAN DER WAALS MOLECULES ${ }^{1}$
}

\author{
G. DELGADO-BARRIO, S. SERNA, S. MIRET-ARTÉS, O. RONCERO, \\ J. CAMPOS-MARTÍNEZ and P. VILLARREAL \\ Instituto de Física Fundamental Centro Mixto C.S.I.C.-U.C.M., C/Serrano 123 \\ 28006 Madrid, Spain
}

(Received 30 May 1991; in final form 15 October 1991)

Different approaches are presented for the study of vibrational predissociation of triatomic van der Waals molecules. These methods are applied to the study of the $\mathrm{He}-I_{2}$ when $I_{2}$ is excited to the $B$ electronic state.

The applicability of these methods is discussed in detail by comparing with "exact" quantum results.

KEY WORDS: Approaches for vibrational predissociation.

\section{INTRODUCTION}

There has been considerable recent interest in the study of van der Waals (vdW) interactions, since these forces have a profound effect on bulk properties, and in the last decade major advances in experimental methods for observing vdW complexes have been done. A great deal of detailed experimental information on intramolecular forces has stimulated theoretical research for calculating intermolecular potential energy surfaces (PES) and for studying the dynamics of these molecules. Various aspects of $\mathrm{vdW}$ molecules have been subject of earlier reviews. ${ }^{1-9}$

VdW molecules are weakly bound complexes of atoms or molecules held together not by chemical bonds but by intermolecular attractions. The vdW forces are roughly $10^{2}-10^{3}$ times weaker than chemical forces, and the dissociation energies are comparable to, or weaker than, $\mathrm{kT}$. The primary characteristics of the vdW molecules are

(a) Low dissociation energies from 10 to $500 \mathrm{~cm}^{-1}$.

(b) Large length of the vdW bond from 2 to $5 \AA$.

(c) Retention of the individual properties of the molecular constituents within the vdW agregate.

The Vibrational Predissociation $(V P)$ of vdW molecules provides interesting features of energy acquisition within a normal chemical bond followed by intramolecular vibrational energy redistribution which results in the fragmentation of the vdW bond. In the vdW molecules the excitation process is well defined and can be well controlled, and the resulting dynamical intramolecular relaxation is a unique example of $V P$ on a single electronic potential energy surface. The

${ }^{1}$ This work was partially supported by a CICYT grant no.: PB87-0272. 
understanding of $V P$ of vdW molecules is relevant for the elucidation of the general features of bond breaking processes in chemical systems.

In this work we shall study the vibrational predissociation of triatomic van der Waals molecules of the type $X \ldots B C$, where $X$ is a rare gas atom and $B C$ is a conventional diatomic molecule. When the chemical bond within the complex is excited we have $X-B C^{*}$ and the energy can flow from the chemical bond to the weak vdW bond and causes its rupture in a time $\tau$

$$
X \ldots B C^{*}(v) \stackrel{\tau}{\rightarrow} B C\left(v^{\prime}<v\right)+X+\Delta E
$$

The fragment $B C$ has lost energy and $X$ flies apart with relative kinetic energy $\Delta E$.

In the vibrational predissociation process, there are three steps:

1. Complex formation, where the $B C$ subunit is in its ground electronic and vibrational state;

2. Optical excitation to a precise vibrational level $v$ in an electronic excited state of $B C$;

3. Predissociation, that results in $X$ and $B C$ fragments, where $B C$ is in a vibrational level $v^{\prime}<v$ of the same electronic state.

The aim of this work is to compare different quasiclassical and quantal approximations to study the $V P$ of three-atomic van der Waals molecules. We apply all of these methods to the $\mathrm{HeI}_{2}$ system that is well known experimentally as well as theoretically.

Levy and co. ${ }^{10}$ have determined the $V P$ lifetimes of $\mathrm{HeI}_{2}\left(B^{3} \mathrm{II}\right)$, produced in a supersonic free expansion, from the linewidths $\Gamma$ of the $R$ branch heads in the fluorescence excitation spectra.

The experimental data of this system are:

1. The dissociation energy $D_{0}$ in the ground vdW state is

$$
13.6 \leqslant D_{0} \leqslant 14.8 \mathrm{~cm}^{-1}
$$

2. The energy difference between the first excited level and the ground vdW state is in the range $[5.47,5.85] \mathrm{cm}^{-1}$.

3. For vibrational excitation of the $\mathrm{I}_{2}$ in the region $v \in[10,45]$ the dependence of $\Gamma$ on the vibrational quantum number, $v$, of $\mathrm{I}_{2}$ follows a superlinear behaviour

$$
\Gamma_{v}=0.555 \times 10^{-4} v^{2}+0.174 \times 10^{-5} v^{3}\left(\mathrm{~cm}^{-1}\right)
$$

The corresponding lifetimes vary from 221 ps at $v=12$ to 38 ps at $v=26$.

In the same energy region the predissociation produces $I_{2}$ essentially in the vibrational state

$$
v^{\prime}=v-1
$$

4. For $v>60$ the most prominent channel is the corresponding to the lost of two vibrational quanta

$$
v^{\prime}=v-2
$$


5. The experiments of $\mathrm{W}$. Sharfin et al. ${ }^{11}$ show a non-quadratic behavior of $\Gamma$ in the region $v \in[50,64]$ with a maximum at $v=57$.

These accurate data call for a confrontation of the theory with the experimental facts. In section 2 we show several quantal and quasiclassical models and in Section 3 they are applied to the $\mathrm{He} \ldots \mathrm{I}_{2}$ system for $23 \leqslant v \leqslant 35$.

\section{METHODOLOGY}

\subsection{Full Three-Dimensional Quantal Model}

In the framework of the first order perturbation theory for electric dipole transitions, the cross section for the excitation from an initial bound state $\left|\Psi_{i}\right\rangle$, with energy $E_{i}$, to a final dissociative state $\left|\Psi_{f E}\right\rangle$ of energy $E$, is given by:

$$
\sigma_{f E \leftarrow i} \propto\left|\left\langle\Psi_{f E}|\mathbf{M} \cdot \mathbf{e}| \Psi_{i}\right\rangle\right|^{2} \quad \text { with } E=E_{i}+\hbar \omega
$$

where $\mathbf{e}$ is the polarization vector of the incident photon, with energy $\hbar \omega, \mathbf{M}$ is the transition dipole moment and $f$ is a collection of quantum numbers which indicates the final quantum state of the fragments after the dissociation.

Within the Born-Oppenheimer approximation, $\left|\Psi_{i}\right\rangle$ and $\left|\Psi_{f E}\right\rangle$ belong to different electronic states, and for the case of triatomic systems of the type $X \ldots B C$ these wavefunctions are solutions of the corresponding Hamiltonians for nuclear motion, which after separation of the center of mass of the whole system, can be written in Jacobi coordinates as:

$$
H=\frac{\hbar^{2}}{2 \mu}\left(-\frac{\partial^{2}}{\partial R^{2}}+\frac{\ell^{2}}{R^{2}}\right)+\frac{\hbar^{2}}{2 m}\left(-\frac{\partial^{2}}{\partial r^{2}}+\frac{\mathbf{j}^{2}}{r^{2}}\right)+U_{B C}(r)+V(R, r, \theta)
$$

where $R$ is the distance between $X$ and the center of mass of $B C, r$ is the internuclear distance for $B C$, and $\theta$ is the angle between the two vectors $\mathbf{r}$ and $\mathbf{R}$, while the $\mu$ and $m$ factors are the corresponding reduced masses. In Eq. (2) $\ell$ and $\mathbf{j}$ are the angular momentum operators (in units of $\hbar$ ) associated with $\mathbf{R}$ and $\mathbf{r}$, respectively. Taking advantage of the weakness of the van der Waals interactions, the total potential for the nuclear motion can be written as a sum of $U_{B C}(r)$, the intramolecular potential interaction for the free diatomic molecule $B C$, and $V$, the intermolecular interaction between the rare gas atom $\mathrm{X}$ and the diatomic molecule $B C$. Thus we have $V \rightarrow 0$ when $R \rightarrow \infty$.

In the neighborhood of resonances the $P$ and $Q$ projection operators are usually introduced, ${ }^{12}$ which verify the relationships:

$$
P+Q=1 ; \quad Q P=P Q=0 ; \quad P^{2}=P
$$

where $P$ projects on a particular set of levels of the total Hamiltonian, which in this case will be the "quasi-discrete" states responsible of the resonant behavior.

The total Hamiltonian can then be written as $H=H_{0}+V$, with:

$$
\begin{gathered}
H_{0}=P H P+Q H Q \\
V=P H Q+Q H P
\end{gathered}
$$


where $H_{0}$ has discrete, $\left|\Phi_{q}\right\rangle$, as well as continuum, $\left|f^{\prime}, E^{\prime}\right\rangle$, solutions which are coupled by the $V$ term as follows:

$$
\begin{gathered}
\left\langle\Phi_{q}|H| f, E\right\rangle=\left\langle\Phi_{q}|V| f, E\right\rangle=V_{f, E} \\
\left\langle\Phi_{q}|H| \Phi_{q^{\prime}}\right\rangle=\left\langle\Phi_{q}\left|H_{0}\right| \Phi_{q^{\prime}}\right\rangle=E_{q} \delta_{q q^{\prime}} \\
\left\langle f, E|H| f^{\prime}, E^{\prime}\right\rangle=\left\langle f, E\left|H_{0}\right| f^{\prime}, E^{\prime}\right\rangle=E \delta_{f f^{\prime}} \delta\left(E-E^{\prime}\right)
\end{gathered}
$$

In the neighborhood of a well isolated resonance, the introduction of the closure relation in the Lippmann-Schwinger equation leads to express the exact dissociative wavefunction $\left|\Psi_{f E}\right\rangle$ in the Fano's formalism, ${ }^{13}$ as:

$$
\left|\Psi_{f E}\right\rangle=a_{f}(E)\left|\Phi_{q}\right\rangle+\sum_{f^{\prime}} \int \mathrm{d} E^{\prime} b_{f^{\prime} E^{\prime}}(f, E)\left|f^{\prime}, E^{\prime}\right\rangle
$$

Near an isolated resonance several situations are possible in a general case: absorption to the zero order discrete state, to the zero order continuum states or to both at the same time. In this last case the total photofragmentation cross-section shows the Fano-Beutler profile corresponding to the interference effects between the transitions to the zero order "discrete" and continuum functions.

For van der Waals systems, the equilibrium distances of the fragmentation coordinate for the initial and final electronic states are very similar i.e., the minima of the van der Waals interaction, $V(R, r, \theta)$, in the fragmentation coordinate $R$ are supposed to do not strongly vary from the initial to the final electronic states. Then, the Frank-Condon factors will strongly favor the optical transition to the excited discrete states, whereas direct excitation to the continuum states will be extremely weak.

Defining the "shift", $\Delta(E)$, and the halfwidth, $\Gamma(E)$, of the resonance as:

$$
\begin{gathered}
\Delta(E)=\sum_{f^{\prime}} \mathscr{P} \int \mathrm{d} E^{\prime} \frac{\left|V_{f^{\prime} E^{\prime}}\right|^{2}}{E-E^{\prime}} \\
\Gamma(E)=\sum_{f^{\prime}} \pi\left|V_{f^{\prime} E}\right|^{2}
\end{gathered}
$$

where $\mathscr{P}$ indicates "principal part", the coefficient of the bound component in Eq. (4), $a_{f}(E)$, takes the form:

$$
a_{f}(E)=\frac{V_{f E}}{\left(E-E_{q}\right)-\Delta(E)-i \Gamma(E)}
$$

In photofragmentation, the predissociation case is characterized by a very long life of the complex before dissociation, i.e., the associated resonance has a very small width. The shift, which is a second order term while the width is of first order, is also small. Thus in the neighborhood of a predissociating resonance the quantities $\Gamma(E), \Delta(E)$ and $V_{f E}$ should be constant as a function of the energy.

Then, near an isolated predissociating resonance the total photoabsorption cross 
section can be approximated by:

$$
\sum_{f} \sigma_{f E \leftarrow i} \propto\left|\left\langle\Phi_{q}|\mathbf{M} \cdot \mathbf{e}| \Psi_{i}\right\rangle\right|^{2} \sum_{f}\left|a_{f}(E)\right|^{2}=\left|\left\langle\Phi_{q}|\mathbf{M} \cdot \mathbf{e}| \Psi_{i}\right\rangle\right|^{2}|a(E)|^{2}
$$

where the first factor of the right-hand side gives the intensity of the absorption and does not depend on energy while the second, with the following Lorentzian form:

$$
\begin{aligned}
|a(E)|^{2} & =\frac{\Gamma / \pi}{\left(E-E_{r}\right)^{2}+\Gamma^{2}} \\
& =\sum_{f}\left|\left\langle\Psi_{f E} \mid \Phi_{q}\right\rangle\right|^{2}
\end{aligned}
$$

gives all the information about the dynamical process, i.e., $E_{r}=E_{q}+\Delta$ and $\Gamma$ independently from the absorption.

The distribution of the final states of the fragments is obtained as:

$$
P_{f}=\frac{\left|\left\langle\Psi_{f E} \mid \Phi_{q}\right\rangle\right|^{2}}{\sum_{f^{\prime}}\left|\left\langle\Psi_{f^{\prime} E} \mid \Phi_{q}\right\rangle\right|^{2}}
$$

$P_{f}$ being constant in the resonance.

The final states of the diatomic fragments are well described in the basis set given by $\left\{\varphi_{v}(r) \cdot \Theta_{j \Omega}^{J M p}(\hat{r}, \hat{R})\right\}$, where the radial functions of $B C, \varphi_{v}(r)$, are solutions of:

$$
\left\{-\frac{\hbar^{2}}{2 m} \frac{\partial^{2}}{\partial r^{2}}+U_{B C}(r)-E_{v}\right\} \varphi_{v}(r)=0
$$

and the "free rotor" functions in the body fixed frame ${ }^{14} \Theta_{j \Omega}^{J M p}(\hat{r}, \hat{R})$, are given by:

$$
\begin{array}{r}
\Theta_{j \Omega}^{J M p}(\hat{r}, \hat{R})=\left[\frac{2 J+1}{8 \pi\left(1+\delta_{\Omega 0}\right)}\right]^{1 / 2}\left\{\begin{array}{r}
D_{M \Omega}^{J *}\left(\phi_{R}, \theta_{R}, 0\right) Y_{j \Omega}(\theta, \phi) \\
\left.\quad+p(-1)^{J} D_{M-\Omega}^{J^{*}}\left(\phi_{R}, \theta_{R}, 0\right) Y_{j-\Omega}(\theta, \phi)\right\}
\end{array}\right.
\end{array}
$$

where $\mathbf{J}=\ell+\mathbf{j}$ is the total angular momentum, $M$ and $\Omega \geqslant 0$ are its projections on the $z$-axis of the space-fixed and body-fixed frames, respectively, and $p= \pm 1$ is the parity under total nuclear coordinates inversion. $D_{M \Omega}^{J^{*}}\left(\phi_{R}, \theta_{R}, 0\right)$ are the Wigner rotational functions and $Y_{j \Omega}(\theta, \phi)$ are the spherical harmonics, $\theta_{R}$ and $\phi_{R}$ being the polar angles specifying the $\mathbf{R}$ vector with respect to the space fixed frame, while $\theta$ and $\phi$ are the polar angles of $\mathbf{r}$ in the body fixed frame. The $\mathbf{j}^{2}$ operator is diagonal in this basis set with eigenvalues $j(j+1)$, while $\ell^{2}$ couples states with different $\Omega$ values:

$$
\begin{aligned}
\left\langle\Theta_{j \Omega}^{J M p}\left|\ell^{2}\right| \Theta_{j^{\prime} \Omega^{\prime}}^{J M p}\right\rangle= & \delta_{j j^{\prime}} \delta_{\Omega \Omega^{\prime}}\left[J(J+1)+j(j+1)-2 \Omega^{2}\right] \\
& -\delta_{j j^{\prime}} \delta_{\Omega \Omega^{\prime} \pm 1}\left[J(J+1)-\Omega^{\prime}\left(\Omega^{\prime} \pm 1\right)\right]^{1 / 2} \\
& \times\left[j(j+1)-\Omega^{\prime}\left(\Omega^{\prime} \pm 1\right)\right]^{1 / 2}
\end{aligned}
$$

The "discrete" function $\left|\Phi_{q}\right\rangle$ is then expanded as:

$$
\Phi_{q}^{J M p}(\mathbf{r}, \mathbf{R})=\sum_{v} \sum_{j, \Omega} \sum_{n} a_{v j \Omega n}^{q} \chi_{n}(R) \varphi_{v}(r) \Theta_{j \Omega}^{J M p}(\hat{r}, \hat{R})
$$


where $\chi_{n}(R)$ is an harmonic oscillator basis set with frequency and equilibrium distance fitted to describe the ground state of the electronic energy surface. The coefficients $a_{v j \Omega n}^{q}$ and the energy $E_{q}$ of $\left|\Phi_{q}\right\rangle$ are obtained by diagonalizing the Hamiltonian matrix in this basis set.

For the "exact" dissociative state, the wavefunction $\left|\Psi_{v j \Omega E}^{J M p-}\right\rangle$ is expanded as:

$$
\Psi_{v j \Omega E}^{J M p-}(\mathbf{r}, \mathbf{R})=\sum_{v^{\prime}} \sum_{j^{\prime} \Omega^{\prime}} \Phi_{E v^{\prime} j^{\prime} \Omega^{\prime}}^{J M p v \Omega^{\prime}-}(R) \varphi_{v^{\prime}}(r) \Theta_{j^{\prime} \Omega^{\prime}}^{J M p}(\hat{r}, \hat{R})
$$

Introduction of Eq. (14) in the time independent Schrödinger equation with the Hamiltonian given in Eq. (2) leads to the close coupled system:

$$
\begin{gathered}
\left\{-\frac{\hbar^{2}}{2 \mu} \frac{\partial^{2}}{\partial R^{2}}+E_{v}+B_{v} j(j+1)-E\right\} \Phi_{E v j \Omega}^{J M p v j \Omega-}(R) \\
=-\sum_{v^{\prime}, j^{\prime}, \Omega^{\prime}}\left\{\delta_{\Omega \Omega^{\prime}}\left\langle\Theta_{j \Omega}^{J M p}\left|V_{v v^{\prime}}\right| \Theta_{j^{\prime} \Omega^{\prime}}^{J M p}\right\rangle \Phi_{E v^{\prime} j^{\prime} \Omega^{\prime}}^{J M p v j \Omega-}(R)\right. \\
\left.+\frac{\left.\delta_{j j^{\prime}} \delta_{v v^{\prime}}\left\langle\Theta_{j \Omega}^{J M}\left|\ell^{2}\right| \Theta_{j^{\prime} \Omega^{\prime}}^{J M p}\right\rangle \Phi_{E v^{\prime} J^{\prime} \Omega^{\prime}}^{J M p v \Omega-}(R)\right\}}{2 \mu R^{2}}\right\}
\end{gathered}
$$

where

$$
\begin{gathered}
B_{v}=\left\langle\varphi_{v}\left|\frac{\hbar^{2}}{2 m r^{2}}\right| \varphi_{v}\right\rangle \\
V_{v v^{\prime}}(R, \theta)=\left\langle\varphi_{v}|V(r, R, \theta)| \varphi_{v^{\prime}}\right\rangle
\end{gathered}
$$

and to obtain the $\Phi_{E v^{\prime} j^{\prime} \Omega^{\prime}}^{J M p j}-(R)$ the usual outgoing asymptotic conditions in terms of the scattering matrix are imposed:

$$
\Psi_{v j \Omega E}^{J M p-}(\mathbf{r}, \mathbf{R}) \underset{R \rightarrow \infty}{\sim} \varphi_{v}(r) \Theta_{j \Omega^{\prime}}^{J M p}(\hat{r}, \hat{R}) \mathrm{e}^{i k_{r i} R}+\sum_{v^{\prime} j^{\prime} \Omega^{\prime}} S_{v j \Omega, v^{\prime} j^{\prime} \Omega^{\prime}}^{*}(E) \varphi_{v^{\prime}}(r) \Theta_{j^{\prime} \Omega^{\prime}}^{J M p}(\hat{r}, \hat{R}) \mathrm{e}^{-i k_{r^{\prime} \gamma^{\prime} R}}
$$

with $k_{v j}=\sqrt{2 \mu\left(E-E_{v}-B_{v} j(j+1)\right)}$.

The potential matrix elements $V_{v v^{\prime}}(R, \theta)$ are usually expanded in terms of Legendre polynomials as:

$$
V_{v v^{\prime}}=\sum_{\lambda} V_{v v^{\prime}}^{\lambda} P_{\lambda}(\cos \theta)
$$

so that the angular matrix elements become:

$$
\left\langle\Theta_{j \Omega}^{J M p}\left|V_{v v^{\prime}}\right| \Theta_{j^{\prime} \Omega^{\prime}}^{J M p}\right\rangle=\delta_{\Omega \Omega^{\prime}} \sum_{\lambda} V_{v v^{\prime}}^{\lambda}\left(\frac{2 j^{\prime}+1}{2 j+1}\right)\left(j^{\prime}, \lambda, j \mid 0,0,0\right)\left(j^{\prime}, \lambda, j \mid \Omega, 0, \Omega\right)
$$

where $\left(j_{1}{ }^{\prime} j_{2}, j_{3} \mid m_{1}, m_{2}, m_{3}\right)$ are the Clebsh-Gordan coefficients.

Usually the resolution of the close coupled equations given by Eq. (15) involves a lot of channels and in what follows some decoupling schemes among the different degrees of freedom will be suggested and discussed. 


\subsubsection{Vibrational diabatic approximation $(V D A)^{15}$}

For low vibrational diatomic states, the "fast" vibrational motion of $B C$ can be diabatically decoupled from the "slow" intermolecular motions of $X \ldots B C$. Therefore it is considered a zero-order Hamiltonian for a given vibrational state:

$$
H_{v}=\frac{\hbar^{2}}{2 \mu}\left(-\frac{\partial^{2}}{\partial R^{2}}+\frac{\ell^{2}}{R^{2}}\right)+B_{v} \mathbf{j}^{2}+E_{v}+V_{v v}(R, \theta)
$$

which has discrete as well as continuum solutions. The stationary states of $H_{v}$ can be expressed as:

$$
{ }^{(0)} \Psi_{v, q}^{J M p}(\mathbf{r}, \mathbf{R})=\varphi_{v}(r) \sum_{j, \Omega} \sum_{n} a_{j \Omega i n}^{v, q} \chi_{n}(R) \Theta_{j \Omega}^{J M p}(\hat{r}, \hat{R})
$$

where the $a_{j \Omega n}^{v, q}$ coefficients and corresponding energies $E_{v q}$ are obtained by diagonalizing the Hamiltonian represented in the basis set described before.

$$
{ }^{(0)} \Psi_{j \Omega E}^{J M p v-}(\mathbf{r}, \mathbf{R})=\varphi_{v}(r) \sum_{j^{\prime} \Omega^{\prime}} \Phi_{E j^{\prime} \Omega^{\prime}}^{J M p j \Omega-}(R) \Theta_{j^{\prime} \Omega^{\prime}}^{J M p}(\hat{r}, \hat{R})
$$

and, after substitution in the time independent Schrödinger equation, the close coupling system of equations expressed in Eq. (15) are reached, where the sum in the right-hand side is restricted to just one $v$ value and it is solved in a similar way.

Those "bound" states become "quasibound" when it is considered their coupling to the continuum states associated to a different $v$ value, at the same energy $E_{v q}$, and the corresponding halfwidth, as given in the "Golden Rule" framework, becomes:

$$
\Gamma_{v q}=\pi \sum_{v^{\prime} \leqslant v, j^{\prime}, \Omega^{\prime}}\left|\left\langle{ }^{(0)} \Psi_{j^{\prime} \Omega^{\prime} E_{r q}}^{J M p v^{\prime}-}|V(r, R, \theta)|^{(\phi)} \Psi_{v, q}^{J M p}\right\rangle\right|^{2}
$$

where $\left|{ }^{(0)} \Psi_{j^{\prime} \Omega^{\prime} E_{r y}}^{J M p w^{\prime}-}\right\rangle$ must be calculated on the energy-shell $E=E_{v q}$.

The final state distribution of the fragments will then be given by:

$$
P_{v^{\prime}, j^{\prime}}^{v q}=\sum_{\Omega^{\prime}} \frac{\pi\left|\left\langle^{(0)} \Psi_{j^{\prime} \Omega^{\prime} E_{r q}}^{J M p v^{\prime}}|V(r, R, \theta)|^{(0)} \Psi_{v, q}^{J M p}\right\rangle\right|^{2}}{\Gamma_{v q}}
$$

\subsubsection{Rotational infinite order sudden approximation $\left(\right.$ RIOSA) ${ }^{16}$}

Due to the anharmonicity of the $B C$ diatomic potential, the higher the diatomic vibration, the closer the different $v$ channels. Then, strong interactions appear between them and the diabatic separation of the intramolecular vibration becomes non appropriate for high $v$ values. In particular, there is a certain $v$ for which the $v-1$ exit channel is closed and then the $v-2$ becomes predominant. In these cases, it appears a strong interaction among quasibound levels with different $v$ quantum number. ${ }^{17}$ For $\mathrm{He} \ldots \mathrm{I}_{2}$ that happens for $v \geqslant 60$.

In these conditions and since in the dissociation the motion along the stretching coordinate, $R$ is faster than the rotation of the $B C$ fragment, the Rotational Infinite Order Sudden Approximation is expected to work very well. In this approximation, 
the total wave-function is expressed as:

$$
{ }^{(0)} \Psi_{v E}^{J M p^{-}}=\Phi_{v E}^{-}(r, R ; \theta) F_{v}^{J M p}(\hat{r}, \hat{R})
$$

where $\Phi_{v E}^{-}(r, R ; \theta)$ are eigenfunctions of the effective Hamiltonian for each value of the internal angle $\theta$ :

$$
H_{\theta}=-\frac{\hbar^{2}}{2 \mu} \frac{\partial^{2}}{\partial R^{2}}-\frac{\hbar^{2}}{2 m} \frac{\partial^{2}}{\partial r^{2}}+U_{B C}+V(R, r ; \theta)
$$

To solve the Schrödinger equation associated to the above Hamiltonian, the following expansion is used:

$$
\Phi_{E}^{v-}(r, R ; \theta)=\sum_{v^{\prime}} \phi_{v^{\prime} E}^{v-}(R ; \theta) \varphi_{v^{\prime}}(r)
$$

that leads to the following set of coupled equations:

$$
\left\{-\frac{\hbar^{2}}{2 \mu} \frac{\partial^{2}}{\partial R^{2}}+E_{v}-E\right\} \phi_{v E}^{v-}(R ; \theta)=-\sum_{v^{\prime}} V_{v v^{\prime}}(R ; \theta) \phi_{v^{\prime} E}^{v-}(R ; \theta)
$$

which is numerically solved by imposing the usual asymptotic conditions.

By solving the close coupled equations at each $\theta$, positions, $E_{r}(\theta)$, half-widths, $\Gamma(\theta)$ and vibrational distributions $P_{v}(\theta)$, are obtained by fitting a Lorentzian function of the energy to the $|a(E)|^{2}$ shape factor (see Eqs. $(8,9)$ ). These quantities can be indexed, approximately, by means of the quantum numbers $v$ and $s$ of the resonance, corresponding to the $r$ and $R$ stretching motions, respectively.

In order to calculate the energies and widths of triatomic predissociating levels it is necessary to include the angular part. Assuming the energy positions of a resonance as a function of $\theta, E_{v, s}(\theta)$, to be the effective potential for the bending motion, the angular part of Eq. $(25), F(\hat{r}, \hat{R})$, is the solution of the equation:

$$
\left\{\frac{\hbar^{2} \ell^{2}}{2 \mu \bar{R}^{2}}+\frac{\hbar^{2} j^{2}}{2 m \bar{r}^{2}}+E_{v, s}(\theta)-E_{v, s, b}\right\} F_{v, s, b}^{J M p}(\hat{r}, \hat{R})=0
$$

where $b$ represents the quantum number associated to the bending motion. In Eq. (29), the fact that discrete wave-functions are mainly sensitive to the details of the potential surface in the region of the well has been used. Hence, $R$ and $r$ are substituted by their equilibrium values, $\bar{R}$ and $\bar{r}$, respectively. Expanding the angular function as:

$$
F_{v, s, b}^{J M p}(\hat{r}, \hat{R})=\sum_{j^{\prime} \Omega^{\prime}} a_{j^{\prime} \Omega^{\prime}}^{v s b} \Theta_{j^{\prime} \Omega^{\prime}}^{J M p}(\hat{r}, \hat{R})
$$

the corresponding coefficients and energies are then obtained by diagonalizing Eq. (29).

The associated halfwidth is then obtained by averaging,

$$
\Gamma_{v, s, b}=\left\langle F_{v, s, b}^{J M p}(\hat{r}, \hat{R})\left|\Gamma_{v, s}(\theta)\right| F_{v, s, b}^{J M p}(\hat{r}, \hat{R})\right\rangle
$$

and the final vibro-rotational state distribution of the diatomic fragment can then be approximated by:

$$
P_{v^{\prime} j^{\prime}}^{v s b}=\sum_{\Omega^{\prime}} a_{j^{\prime} \Omega^{\prime}}^{v s b} \sum_{j^{\prime \prime} \Omega^{\prime \prime}} a_{j^{\prime} \Omega^{\prime \prime}}^{v s b}\left\langle\Theta_{j^{\prime} \Omega^{\prime}}^{J M p}(\hat{r}, \hat{R})\left|P_{v}(\theta)\right| \Theta_{j^{\prime \prime} \Omega^{\prime \prime}}^{J M p}(\hat{r}, \hat{R})\right\rangle
$$




\subsubsection{Vibrational diabatic and rotational infinite order sudden approximation} $(\text { VIDRIOSA })^{18}$

The two approaches described above can be applied at the same time to produce a more decoupled scheme, in which, again, there will be discrete and continuum zero order functions. The stationary states are expressed as:

$$
{ }^{(0)} \Psi_{v, s, b}^{J M p}(\mathbf{r}, \mathbf{R})=\varphi_{v}(r) \phi_{v, s}(R ; \theta) F_{v, s, b}^{J M p}(\hat{r}, \hat{R})
$$

where, as used before, $v, s$ and $b$ are the quantum numbers associated with the diatomic stretching, van der Waals stretching and bending motions, respectively. The functions $\phi_{v, s}(R ; \theta)$ are discrete solutions, at each $\theta$ value, of the Schrödinger equation:

$$
\left\{-\frac{\hbar^{2}}{2 \mu} \frac{\partial^{2}}{\partial R^{2}}+V_{v v}(R ; \theta)-W_{v, s}(\theta)\right\} \phi_{v, s}(R ; \theta)=0
$$

where the eigenvalues $W_{v, s}(\theta)$ will act as an effective potential for the bending motion. Then, neglecting the effect of the angular momentum operator on the $\phi_{v, s}(R ; \theta)$ function, i.e.:

$$
\begin{aligned}
& \ell^{2} \phi_{v, s}(R ; \theta) F_{v, s, b}^{J M p}(\hat{r}, \hat{R})=\phi_{v, s}(R ; \theta) \ell^{2} F_{v, s, b}^{J M p}(\hat{r}, \hat{R}) \\
& \mathbf{j}^{2} \phi_{v, s}(R ; \theta) F_{v, s, b}^{J M p}(\hat{r}, \hat{R})=\phi_{v, s}(R ; \theta) \mathbf{j}^{2} F_{v, s, b}^{J M p}(\hat{r}, \hat{R})
\end{aligned}
$$

it results that the $F_{v, s, b}^{J M p}(\hat{r}, \hat{R})$ are solution of the equation:

$$
\left\{\frac{\hbar^{2}}{4 \mu}\left[\ell^{2}, \bar{R}_{v, s}^{-2}(\theta)\right]+B_{v} \mathbf{j}^{2}+W_{v, s}(\theta)-E_{v, s, b}\right\} F_{v, s, b}^{J M p}(\hat{r}, \hat{R})=0
$$

where $[A, B]=A B+B A$ is the anticommutator introduced to symmetrize the approximated Hamiltonian and:

$$
\bar{R}_{v, s}^{-2}(\theta)=\left\langle\phi_{v, s}(R ; \theta)\left|\frac{1}{R^{2}}\right| \phi_{v, s}(R ; \theta)\right\rangle
$$

The zero order continuum solutions are written, in the RIOSA frame, as:

$$
{ }^{(0)} \Psi_{v, j, \Omega, E}^{J M p-}(\mathbf{r}, \mathbf{R})=\varphi_{v}(r) \phi_{v, E}^{-}(R ; \theta) \Theta_{j \Omega}^{J M p}(\hat{r}, \hat{R})
$$

where $\phi_{v, E}^{-}(R ; \theta)$ are continuum solutions of Eq. (34) fulfilling similar outgoing asymptotic conditions than (Eq. (17)), except the absence of angular functions.

Taking now into account the coupling in the first order "Golden Rule" scheme, the halfwidth of the quasibound level labeled by the $(v, s, b)$ quantum numbers is:

$$
\Gamma_{v, s, b}=\pi \sum_{v^{\prime}, j^{\prime}, \Omega^{\prime}}\left|\left\langle\Theta_{j^{\prime} \Omega^{\prime}}^{J M p}\left|\left\langle V_{v^{\prime} v}(\theta)\right\rangle\right| F_{v, s, b}^{J M p}\right\rangle\right|^{2}
$$

where:

$$
\left\langle V_{v^{\prime} v}(\theta)\right\rangle=\left\langle\phi_{v^{\prime}, E}^{-}(R ; \theta)\left|V_{v^{\prime} v}(R, \theta)\right| \phi_{v, s}(R ; \theta)\right\rangle
$$

are the relevant potential coupling terms which usually are expanded in Legendre 
polynomials (Eq. (18)) so that the integrals involved in Eq. (39) become analytical (Eq. (19)).

The final state distribution of $B C$ will be given by:

$$
P_{v^{\prime}, j^{\prime}}=\pi \sum_{\Omega^{\prime}} \frac{\left|\left\langle\Theta_{j^{\prime} \Omega^{\prime}}^{J M p}\left|\left\langle V_{v^{\prime} v}(\theta)\right\rangle\right| F_{v, s, b}^{J M p}\right\rangle\right|^{2}}{\Gamma_{v, s, b}}
$$

\subsubsection{Vibrational and rotational diabatic approximation $(V R D A)^{19}$}

Finally, the "crudest" approach considered here neglects the coupling among channels with different $(v, j)$ values. The zero order wavefunctions are then written as:

$$
{ }^{(0)} \Psi_{v j \Omega}^{J M} p(\mathbf{r}, \mathbf{R})=\phi_{v j \Omega v}(R) \Theta_{j \Omega}^{J M p}(\hat{r}, \hat{R}) \varphi_{v}(r)
$$

where the functions $\phi_{v j \Omega v}(R)$ are the discrete and continuum solutions of:

$$
\left\{-\frac{\hbar^{2}}{2 \mu} \frac{\partial^{2}}{\partial R^{2}}+\left\langle\Theta_{j \Omega}^{J M p}\left|V_{v v}(R, \theta)\right| \Theta_{j \Omega}^{J M p}\right\rangle+E_{v}+B_{v} j(j+1)-E\right\} \phi_{v j \Omega v}(R)=0
$$

where $v$ is an integer for discrete solutions and the energy for the continuum solutions.

As in the VD approach, the halfwidth and final state distributions will be obtained in a similar way as those given by Eqs. (23) and (24), respectively.

The description of the bound states can be very easily improved by taking into account the configuration interaction among the zero order discrete functions, thus obtaining the $\Phi_{q}^{J M p}$ functions given by Eq. (13), but still neglecting the coupling among the continuum solutions (CIVRDA).

\subsection{Quasiclassical Model}

The classical trajectory method has been extensively applied in reactive and inelastic collisions. ${ }^{20}$ It consists in solving the relevant Hamilton equations once the initial conditions (coordinates and momenta of every particle) have been established.

For a $X \ldots B C$ triatomic system the elimination of the coordinates of one of the atoms ( $B$ in this case), in the center of mass system, leads to the Hamiltonian:

$$
H=\frac{m_{B}+m_{C}}{2 m_{X} M} P_{X}^{2}+\frac{m_{B}+m_{X}}{2 M \cdot m_{C}} P_{C}^{2}-\frac{P_{X} \cdot P_{C}}{M}+V
$$

where the $P$ 's are the conjugate momenta to the coordinates, $M$ is the total mass, and $V$ is the potential energy.

The first initial coordinate is selected $r$ according to the distribution of a nonrotating Morse oscillator: ${ }^{21}$

$$
r=\bar{r}-\frac{1}{\alpha} \ln \left\{\frac{-2 a}{b+\left(b^{2}-4 a c\right)^{1 / 2} \sin (2 \pi s)}\right\}
$$

where $a=E-D, b=2 D, c=-D$ and $s$ is a random number uniformly distributed in the range $[0,1]$. Here $E$ is the energy of the vibrational quantum level of $B C$ 
relative to the minimum of the well and $D, \alpha, \bar{r}$ are the Morse parameters of this diatomic molecule.

The angle $\theta$ is randomly chosen according to a uniform distribution in the range $[0, \pi / 2]$ or $[0, \pi]$, and then a random length $R$ is selected in the range $[3,9] \AA$ (also uniformly), where the van der Waals potential well is typically located. In order to calculate the atom-diatom relative momentum, the quantities $r, R$ and $\theta$ must satisfy the following unequality:

$$
E_{v, s, b}-V(R, r, \theta) \geqslant 0
$$

where $E_{v, s, b}$ is the van der Waals energy. If this expression is not fulfilled, the random selection o $\theta$ and $R$ is iterated while the value of $r$ is kept fixed. After the initial coordinates are determined, the associated momenta can be straightforward calculated. ${ }^{22}$

For each trajectory, the duration of the complex is calculated as the elapsed time from the beginning till the last turning point $R$ was reached. Hence, for each starting diatomic vibrational level $v$, the distribution of trajectories was fitted to an exponential law ${ }^{23}$

$$
N_{N D}=N_{T} \exp (-t / \tau)
$$

where $N_{T}$ is the total number of trajectories, $N_{N D}$ is the number of nondissociated trajectories at time $t$ and $\tau$ is the lifetime of the complex. Thus, the half-width is calculated as

$$
\Gamma=\frac{\hbar}{2 \tau}
$$

A plot of $\ln \left(N_{N D} / N_{T}\right)$ versus time $t$ can be useful in order to determine the reliability of the assumed exponential law of decay. A linear fitting of this function provides the lifetime and, by means of Eq. (48), the linewidth.

After a box quantization, by dividing the number of trajectories leading to a diatomic rotational level $j$ by the total number of them, the probabilities $P_{j}$ of the exiting diatomic rotational channels are calculated.

\section{THE He... $\mathrm{I}_{2}$ SYSTEM}

The full potential energy surface is represented by a simple addition of pairwise atom-atom interactions

$$
W(R, r, \theta)=U_{\mathrm{I}_{2}}(r)+V(R, r, \theta)=U_{\mathrm{I}_{2}}(r)+V_{\mathrm{I}-\mathrm{He}}\left(R_{1}\right)+V_{1-\mathrm{He}}\left(R_{2}\right)
$$

each one of them being described by Morse functions of the type

$$
D\left\{e^{-2 \alpha\left(X-X_{e}\right)}-2 \mathrm{e}^{-\alpha\left(X-X_{e}\right)}\right\}
$$

where: $X=r, R_{1}$ or $R_{2}$ and the corresponding parameters-well depth $D$, characteristic inverse length $\alpha$, and equilibrium bond length $X_{e}$-are listed in Table 1 . 
Table 1 Morse parameters for the atom-atom potentials and masses used for the present calculations

\begin{tabular}{lcll}
\hline & $D\left(\mathrm{~cm}^{-1}\right)$ & $\alpha\left(\AA^{-1}\right)$ & $X_{e}(\AA)$ \\
\hline $\mathrm{I}-\mathrm{I}$ & 4391 & 1.834 & 3.016 \\
$\mathrm{I}-\mathrm{He}$ & 18 & 1.14 & 4 \\
\hline
\end{tabular}

$m_{1}=126.9045, m_{\mathrm{He}}=4.0026$.

Table 2 Dissociation energies (and halfwidths) as a function of $v$ for different quantal approaches (in $\mathrm{cm}^{-1}$ )

\begin{tabular}{lccccc}
\hline$v$ & 23 & 26 & 29 & 32 & 35 \\
\hline "Exact" & -19.594 & -19.531 & & & -19.305 \\
& $(0.037)$ & $(0.050)$ & & & $(0.1240)$ \\
VDA & -19.621 & -19.562 & -19.498 & -19.427 & -19.349 \\
& $(0.038)$ & $(0.053)$ & $(0.974)$ & $(0.097)$ & $(0.129)$ \\
RIOSA & $(0.039)$ & $(0.052)$ & & & \\
VIDRIOSA & -19.914 & -19.865 & -19.812 & -19.753 & -19.687 \\
& $(0.043)$ & $(0.059)$ & $(0.080)$ & $(0.108)$ & $(0.144)$ \\
CIVRDA & -19.621 & -19.562 & -19.498 & -19.427 & -19.349 \\
& $(0.006)$ & $(0.007)$ & $(0.009)$ & $(0.010)$ & $(0.011)$ \\
\hline
\end{tabular}

(1) Taken from Ref. 16.

In Eq. (49), the individual I - He distances are trivially expressed in Jacobi coordinates through the geometric relations

$$
R_{i}=\sqrt{R^{2}+r^{2} / 4+(-1)^{i} R r \cos \theta} ; \quad i=1,2
$$

The $V P$ process is not very sensitive to long range interactions because is essentially determined by bound-continuum couplings. It has been shown ${ }^{24-27}$ that representing the individual interactions by Morse functions provides a good description of the potential around the minimum of the surface. The Morse parameters, $D, \alpha$ and $X_{e}$, are usually obtained from a fitting of the available experimental data (dissociation energy, lifetime, final rotational distribution, ...).

Here, we study the $V P$ of $\mathrm{He} \ldots \mathrm{I}_{2}$ when the diatomic fragment is initially excited to a vibrational level $v$ in the range $23 \leqslant v \leqslant 35$. As it has been mentioned before, in this region the experimental halfwidth, $\Gamma$, shows a superlinear behavior as a function of $v$ and the anharmonicity effects are not very strong so that the approximations described above should be well adapted.

In Table 2, dissociation energies and halfwidths as functions of $v$ are shown for different quantal approaches. The "exact" results are obtained by solving Eq. (20) accounting for 75 channels $(v-1, v$ and $v+1$ and $j=0,2, \ldots, 48$, with $J=0)$ using a Fox-Godwin-Numerov integrator, ${ }^{28}$ starting at $1 \AA$ with a step of $\sim 0.01 \AA$ and 2000 points in the grid. The $|a(E)|^{2}$ energy function should follow a typical Lorentzian behavior (see Eq. (13)).

In Figure 1, the "exact" numerical points obtained from the resolution of Eq. (15), 


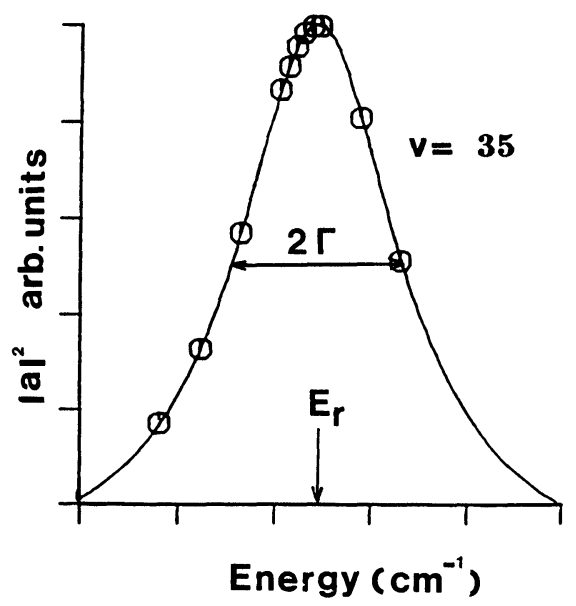

Figure $1|a(E)|^{2}$ function (in $\left.\mathrm{cm}^{1}\right)$ for the resonance corresponding to $(v=35, s=0, b=0)$ approximated quantum numbers.

and the analytical Lorentzian fit for the resonance corresponding to the first van der Waals level of $v=35$ are depicted.

VDA results are very similar to the "exact" ones. The small discrepancies increase with $v$ and for the halfwidth, $\Gamma$, which is a more sensitive magnitude than the energy, the error varies from $\sim 3 \%$ for $v=23$ to $\sim 9 \%$ for $v=35$. The coupling among different $v$ channels, neglected in the VD approximation, increases with $v$ since, due to the anharmonicity, the energy gap between neighboring channels decreases.

The behavior of the VDA and "exact" results are very similar. As it is expected, the higher the vibrational state of the $B C$ fragment, the wider the resonance. This is simply explained in terms of the "energy gap law":29 because of the anhamonicity of the intramolecular potential, the kinetic energy of the fragments decreases when $v$ is increased; the corresponding continuum wavefunction presents less oscillations giving rise to a better overlap with the "stationary' state.

The RIOSA results agree very well with the "exact" ones showing the correct behavior. Now the coupling among $v$ channels is taken into account and the error is expected to be a constant function of $v$, being $\sim 5 \%$ for $v=23$ and for $v=26$. Therefore, for higher intramolecular vibrational excitations, this approach should be still well adapted, in contrast with the VDA neglecting those couplings among different $v$ channels.

This method has also been applied to study the $V P$ of $\mathrm{Ne} \ldots \mathrm{I}_{2}{ }^{17}$ for $28 \leqslant v \leqslant 34$, i.e., the energetic region for which the $v-1$ exit channel becomes closed. The halfwidth shows a bimodal shape as a function of $v$, with one maximum for $v=30$ and another for $v=33$. If there were no vibrational couplings only one peak would be present, corresponding to the $v$ value from which the $v-1$ exit channel becomes closed. When the vibrational couplings are taken into account, it appears a strong interaction among "stationary" states with different $v$ quantum number and a mixing takes place. 
Then the $V P$ process can be seen as a combination of $v \rightarrow v-2$ and $v-1 \rightarrow v-2$ decays, and it is the last one, corresponding to the loss of one vibrational quantum, the responsible for the enhancement. In this case the VDA method is no longer suitable because does not correctly describe the couplings.

The VIDRIOSA method is a combination of VDA and RIOSA and only can be applied when the two precedent methods are good enough. In Table 2 it can be seen that the difference among the VIDRIOSA and the "exact" results is of about $15 \%$ and constant with $v$ and always overestimates the widths. However the behavior is correct and gives a very good description of the problem with a very low computational effort. As in the VDA, it will not give good results when the vibrational couplings become important.

The crudest approach, CIVRDA, describes correctly the energy resonance positions but provides wrong values for the widths, of the order of 6 times smaller than the "exact" ones. That is reasonable since if the process is correctly explained by freezing the angular motion then to use pure rotational states of the diatomic fragment should give a poor description.

The final state distribution of the fragments provides a deep information about the dissociation and, in particular, the rotational branching ratio gives information about the anisotropy of the potential. In the present case the diatomic fragment is homonuclear, the equilibrium configuration is T-shaped and the $I_{2}$ fragment is very heavy as compared with the He atom. Then, after the fragmentation, the $I_{2}$ is unable to present a high rotational excitation. Unfortunately, the rotational diatomic constant, $B_{v}$, is very small $\left(\sim 0.023 \mathrm{~cm}^{-1}\right)$ and there are not experimental data available about final rotational distributions of the $I_{2}$ fragment. However we shall compare the results obtained through the different methods because the final rotational distribution is a very sensitive magnitude.

For systems with an homonuclear diatomic fragment lighter ${ }^{25,30}$ than $I_{2}$, higher rotational excitation is obtained but the maximum of the distribution remains at low $j$ values. That is because when the two fragments fly appart the atom directly "pushes" on the center of mass of the homonuclear diatomic fragment and an amount of energy can be transfered into rotation, keeping the rotational distribution of the low rotational excited quasibound level of the van der Waals complex.

In contrast, for heteronuclear systems as $\mathrm{He} \ldots \mathrm{ICl}^{27}$ and $\mathrm{Ne} \ldots \mathrm{ICl}^{26}$ the diatomic fragments do present very high rotational excitation. In these two systems the rare gas atom lies close to the chlorine end in the equilibrium configuration so that when the fragmentation takes place the $X$ atom "push" the lighter part of the diatomic fragment producing highly rotational excited $B C$ molecules. When changing from $\mathrm{He}$ to $\mathrm{Ne}$ the maximum of the rotational distribution shifts to higher $j$ values due to mass effect. ${ }^{31}$

The final rotational state distributions on $v-1$ are nearly constant with the energy in a resonance. In Figure 2 the "exact" rotational distribution of the $\mathrm{I}_{2}$ product, coming from the fragmentation of the first van der Waals level for $v=23$, is shown together with the decomposition of the "stationary" state (see Eq. (13)) on the "free" rotor basis of the diatom and the quasiclassical results of Ref. (32).

In order to apply the quasiclassical model the relevant Hamilton equations have 


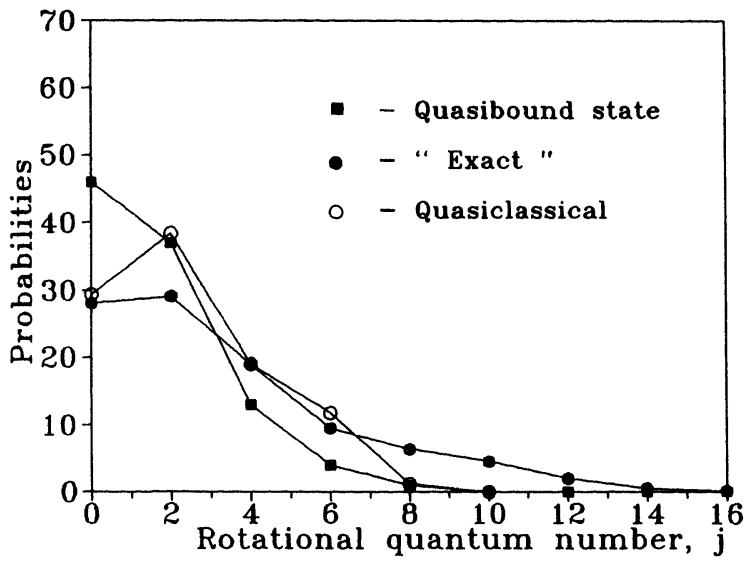

Figure 2 Final rotational distribution of the $I_{2}$ fragment when the complex is initially excited to $v=23$. It is also presented the decomposition of the initial "quasibouns" state.

been numerically solved by using an Adams-Moulton integrator initiated by the fourth order Runge-Kutta-Gill integrator. ${ }^{32,33}$ The convergency for each magnitude has been found to be very dependent on the total time of each trajectory. For these trajectories with a total time of $50 \mathrm{ps}$ the convergent time step was of $0.4 \times 10^{-3} \mathrm{ps}$. In order to determine the initial conditions we have assumed $J=0$ and a starting diatomic rotational energy corresponding to $j=0$, which is the most probable quantum rotational state of $I_{2}$ within the complex. An exact treatment should use a distribution for each of these magnitudes. However, the experimental temperature associated with the distribution over $J$ is very low. ${ }^{10}$ Typically, 100 trayectories have been taken into account. ${ }^{32}$ Due to the poor statistics, the low rotational levels are somehow overestimated, as can be seen in Figure 2. Both, "exact" and quasiclassical, are shifted one rotational quantum as compared with the expansion of the "initial" state, showing that in the fragmentation there is a small angular momentum transfer, as it was predicted above.

In Figure 3, the rotational distributions for $v=23$, obtained through several quantal approaches, are shown. The "exact" and VDA results are the same in this scale, showing the suitability of such an approach in this $v$ range.

The VIDRIOSA overestimates the probability of finding large $j$ values and reduces that of low $j$, keeping the general structure of the "exact" results. The RIOSA can be considered as a combination of the centrifugal and the energy sudden approximations. Population of higher $j$ values, as compared with the "exact" calculation, is a direct consequence of neglecting the centrifugal barrier in the standard RIOSA. States with large $j$ values $(\ell=j$ for $J=0)$ are more easily accessible and the probability is then increased. On the other hand, for low $j$ 's where the centrifugal barrier is not very important, the kinetic energies are increased so that the final probabilities for these states decrease, in agreement with the energy gap law.

The CIVRDA results are however in disagreement with the "exact" ones. While 


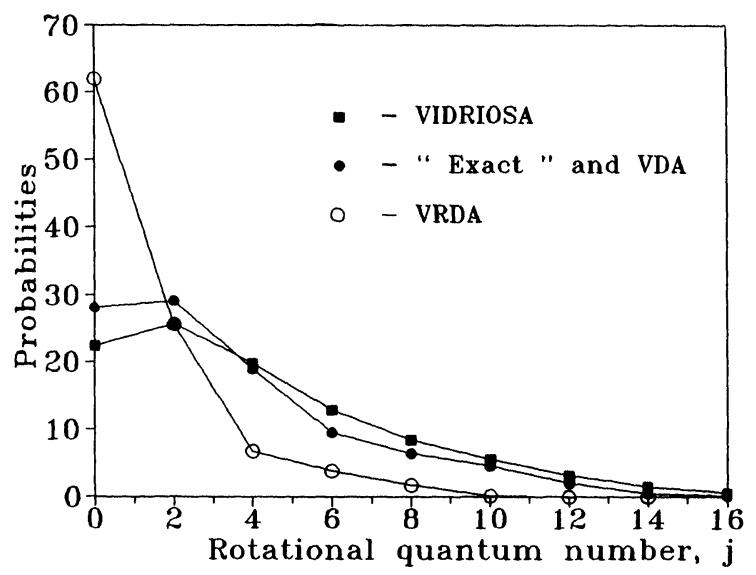

Figure 3 Final rotational distribution of the $I_{2}$ fragment obtained with different quantal methods when the complex is excited to $v=23$.

the maximum of the final rotational state distribution is shifted to higher values of $j$ for the "exact" model as compared with the "initial" rotational distribution (see Figure 2), the opposite case happens in this approach: the probability of finding $j=0$ is overestimated as a result of a transfer from the "initial" angular momentum towards the relative motion of the fragments.

In Tables 3, 4 and 5 the approximated quantal final rotational distributions of the $I_{2}$ fragments as a function of the initial vibrational quantum number are presented. In

Table 3 Final rotational distribution of $I_{2}$ obtained with the VDA method

\begin{tabular}{|c|c|c|c|c|c|c|c|c|c|c|c|}
\hline$v$ & $P_{0}$ & $P_{2}$ & $P_{4}$ & $P_{6}$ & $P_{8}$ & $P_{10}$ & $P_{12}$ & $P_{14}$ & $P_{16}$ & $P_{18}$ & $P_{20}$ \\
\hline 23 & 28.45 & 30.11 & 18.90 & 9.39 & 6.21 & 4.39 & 1.95 & 0.51 & 0.09 & 0.01 & 0.002 \\
\hline 26 & 28.48 & 30.00 & 18.86 & 9.59 & 6.34 & 4.30 & 1.84 & 0.48 & 0.09 & 0.01 & 0.002 \\
\hline 29 & 28.70 & 29.94 & 18.73 & 9.77 & 6.46 & 4.16 & 1.70 & 0.44 & 0.08 & 0.01 & 0.002 \\
\hline 32 & 28.70 & 29.80 & 18.71 & 10.01 & 6.58 & 4.07 & 1.61 & 0.41 & 0.08 & 0.01 & 0.001 \\
\hline 35 & 28.67 & 29.64 & 18.71 & 10.27 & 6.71 & 3.97 & 1.53 & 0.40 & 0.09 & 0.02 & 0.001 \\
\hline
\end{tabular}

Table 4 Final rotational distributions of $\mathrm{I}_{2}$ for the first vdW level as a function of $v$ obtained with the VIDRIOSA method

\begin{tabular}{lllllllllll}
\hline$v$ & $P_{0}$ & $P_{2}$ & $P_{4}$ & $P_{6}$ & $P_{8}$ & $P_{10}$ & $P_{12}$ & $P_{14}$ & $P_{16}$ & $P_{18}$ \\
\hline 23 & 22.37 & 25.63 & 19.75 & 12.85 & 8.36 & 5.52 & 3.19 & 1.50 & 0.57 & 0.19 \\
26 & 22.53 & 25.67 & 19.65 & 12.80 & 8.36 & 5.51 & 3.17 & 1.48 & 0.57 & 0.19 \\
29 & 22.71 & 25.71 & 19.55 & 12.75 & 8.37 & 5.50 & 3.14 & 1.46 & 0.56 & 0.18 \\
32 & 22.91 & 25.76 & 19.44 & 12.70 & 8.37 & 5.48 & 3.10 & 1.44 & 0.55 & 0.18 \\
35 & 23.13 & 25.81 & 19.33 & 12.64 & 8.37 & 5.45 & 3.06 & 1.41 & 0.54 & 0.18 \\
\hline
\end{tabular}


Table 5 Final rotational distribution of $I_{2}$ obtained with the CIVRDA method

\begin{tabular}{|c|c|c|c|c|c|c|c|c|c|c|c|}
\hline$v$ & $P_{0}$ & $P_{2}$ & $P_{4}$ & $P_{6}$ & $P_{8}$ & $P_{10}$ & $P_{12}$ & $P_{14}$ & $P_{16}$ & $P_{18}$ & $P_{20}$ \\
\hline 23 & 61.98 & 25.57 & 6.77 & 3.82 & 1.70 & 0.16 & $0.16(-3)$ & $0.15(-3)$ & & & \\
\hline 26 & 65.35 & 22.81 & 6.63 & 3.68 & 1.42 & 0.193 & $0.7(-5)$ & $0.1(-3)$ & & & \\
\hline 29 & 68.49 & 20.33 & 6.44 & 3.51 & 1.17 & 0.64 & 0.0002 & & & & \\
\hline 32 & 71.40 & 18.10 & 6.21 & 3.31 & 0.94 & 0.04 & 0.0006 & & & & \\
\hline 35 & 74.11 & 16.09 & 5.95 & 3.08 & 0.74 & 0.02 & 0.0009 & & & & \\
\hline
\end{tabular}

Table 6 Final rotational distribution of $\mathrm{I}_{2}$ for the first van der Waals level as a function of $v$ obtained by the "exact" method

\begin{tabular}{llllllllllll}
\hline$v$ & $P_{0}$ & $P_{2}$ & $P_{4}$ & $P_{6}$ & $P_{8}$ & $P_{10}$ & $P_{12}$ & $P_{14}$ & $P_{16}$ & $P_{18}$ & $P_{20}$ \\
\hline 23 & 28.10 & 29.10 & 18.91 & 9.49 & 6.37 & 4.54 & 2.04 & 0.54 & 0.10 & 0.01 & 0.002 \\
26 & 28.04 & 29.90 & 18.90 & 9.72 & 6.52 & 4.48 & 1.94 & 0.51 & 0.10 & 0.01 & 0.002 \\
35 & 27.72 & 29.25 & 18.96 & 10.55 & 7.00 & 4.26 & 1.69 & 0.45 & 0.10 & 0.02 & 0.001 \\
\hline
\end{tabular}

Table 7 Final rotational distributions of $\mathrm{I}_{2}$ obtained with the quasiclassical method $^{32}$

\begin{tabular}{lllllll}
\hline$v$ & $P_{0}$ & $P_{2}$ & $P_{4}$ & $P_{6}$ & $P_{8}$ & $P_{10}$ \\
\hline 23 & 29.4 & 38.4 & 19.0 & 11.8 & 1.2 & \\
26 & 27.3 & 40.5 & 20.1 & 10.7 & 1.1 & \\
29 & 22.4 & 30.4 & 31.2 & 11.0 & 4.0 & 0.8 \\
32 & 25.0 & 35.4 & 19.0 & 14.8 & 3.8 & 1.6 \\
35 & 26.0 & 28.2 & 23.2 & 14.9 & 6.6 & 1.8 \\
\hline
\end{tabular}

principle, when $v$ increases the kinetic energy decreases and then the rotational distribution is shifted to lower $j$ values because the available energy decreases. However, the "exact" and quasiclassical results shown in Tables 6 and 7 do not show that regular behavior. The vibrational couplings have been included in the two methods, approximately in the quasiclassical case ${ }^{32}$ and with few number of trajectories. Therefore, we can conclude that the vibrational couplings strongly affect the rotational distribution of the diatomic fragment.

\section{CONCLUDING REMARKS}

The $V P$ process provides a valuable information about the energy potential surface of the system and, in particular, we have stressed the important role of the final rotational distribution of the diatomic fragment to describe its anisotropy. Since, in general, it is very difficult (and in practice impossible) to obtain the interacting potentials for heavy systems from "first principle" methods, it is important to develop approximate dynamical methods to invert the potential from experimental data, 
determining at what extent they might be used. Here we have described different approximations and discussed their applicability.

In addition, as long as the van der Waals interactions are very weak, the potential energy surface may be described as a sum of atom-atom for no very large systems, where three-body interactions are expected to remain small enough. Hence, the study of the dynamics of bigger van der Waals clusters, as $\mathrm{He}_{n} \ldots \mathrm{I}_{2} \ldots \mathrm{Ne}_{m}{ }^{34}$ and $\mathrm{Ne}_{2} \ldots \mathrm{Cl}_{2},{ }^{35}$ for which experimental data are available, results feasible. These clusters present new general phenomena, as the multifragmentation closely related to reactivity; they are systems of intermediate size providing an ideal case to study the transition between diluted and condensed phases as well as between quantal and classical behaviors.

\section{References}

1. A. van der Avoid, P. E. S. Wormer, F. Nulder and R. M. Berns, Top. Cur. Chem. 93, 1 (1980).

2. J. H. van Lenthe, J. G. C. M. van Duijneveldt-van de Rijdt and F. B. van Duijneveldt, Adv. Chem. Phys. 69, 521 (1987)

3. A. D. Buckingham, P. W. Fowler and J. M. Hutson, Chem. Rev., 88, 963 (1988).

4. J. A. Beswick and J. Jortner, Adv. Chem. Phys. 47, 363 (1981).

5. D. H. Levy, Adv. Chem. Phys. 47, 323 (1981).

6. K. C. Janda, Adv. Chem. Phys. 60, 201 (1985).

7. Faraday Discuss. Chem. Soc. 73 (1982).

8. "Structure and Dynamics of Weakly Bound Molecular Complexes", NATO ASI Series C: Mathematical and Physical Sciences, Vol. 212, Reidel, Dordrecht, 1987.

9. "Dynamics of Polyatomic van der Waals Complexes", Vol. B, Edited by N. Halberstadt and K. C. Janda, Plenum Publishing Corporation, New York, 1989 (in press).

10. R. E. Smalley, D. H. Levy and L. Wharton, J. Chem. Phys. 64, 3266 (1976); M. S. Kim, R. E. Smalley, L. Wharton and D. H. Levy, J. Chem. Phys. 65, 1216 (1976); K. E. Johnson, L. Wharton and D. H. Levy, J. Chem. Pjys. 69, 2719 (1978); W. Sharfin, K. E. Johnson, L. Wharton and D. H. Levy, J. Chem. Phys. 71, 1283 (1979); J. A. Blazy, B. M. Dekoven, T. D. Russell and D. H. Levy, J. Chem. Phys. 72, 2439 (1980).

11.W. Sharfin, P. Kroger and S. C. Wallace, Chem. Phys. Lett., 85, 81 (1982).

12. L. Mower, Phys. Rev. 142, 799 (1966).

13. U. Fano, Phys. Rev. 124, 1866 (1961).

14. R. T. Pack, J. Chem. Phys. 60, 633 (1974); G. C. Schatz and A. Kupperman, J. Chem. Phys. 65, 4642 (1976); R. N. Zare, "Angular Momentum: Understanding Spatial Aslects in Chemistry and Physics", J. Wiley, New York, 1988.

15. J. A. Beswick and G. Delgado-Barrio, J. Chem. Phys. 73, 3653 (1980).

16. G. Delgado-Barrio, P. Mareca, P. Villarreal, A. M. Cortina and S. Miret-Artés, J. Chem. Phys. 84, 4268 (1968); G. Delgado-Barrio, P. Villarreal, P. Mareca and J. A. Beswick, Int. J. Quantum Chem. 27, 173 (1985).

17. O. Roncero, J. Campos-Martinez, A. Cotina, P. Villarreal and G. Delgado-Barrio, Chem. Phys. Lett. 148, 62 (1988).

18. M. Aguado, P. Villarreal, G. Delgado-Barrio, P. Mareca and J. A. Beswick, Chem. Phys. Lett. 102, 227 (1983).

19. J. A. Beswick and A. Requena, J. Chem. Phys., 73, 4347 (1980).

20. D. L. Bunker and N. C. Blais, J. Chem. Phys. 41, 2377 (1964); D. L. Bunker, "Methods in Computational Physics" (Academic, New York, 1971), Vol. 10, p. 287; M. D. Pattengil in "Atomic-Molecule Collision Theory", Edited by R. B. Bernstein (Plenum, New York, 1979); R. N. Porter and L. M. Raff, in "Modern Theoretical Chemistry", Edited by W. H. Miller (Plenum, New York, 1976), Vol. 1, Part B. 
21. R. N. Porter, L. M. Raff and W. H. Miller, J. Chem. Phys. 63, 2214 (1975).

22. S. B. Woodruff and D. L. Thompson, J. Chem. Phys. 71, 376 (1979).

23. K. P. Huber and G. Herzber, "Molecular Spectra and Molecular Structures: IV Constants of Diatomic Molecules", van Nostrand Reinhold Co. (1979).

24. E. De Pablo, M. S. Guijarro, P. Villarreal, P. Mareca and G. Delgado-Barrio, An. Fis. A80, 210 (1984); E. de Pablo, S. Miret-Arte's, P. Mareca, P. Villarreal and G. Delgado-Barrio, J. Mol. Struct. 142, 505 (1986).

25. N. Halberstadt, J. A. Beswick and K. C. Janda, J. Chem. Phys. 87, 3966 (1987); N. Halberstadt, O. Roncero and J. A. Beswick, Chem. Phys. 129, 83 (1989).

26. O. Roncero, J. A. Beswick, N. Halberstadt, P. Villarreal and G. Delgado-Barrio, J. Chem. Phys. 92, 3348 (1990).

27. R. L. Waterland, M. Lester and N. Halberstadt, J. Chem. Phys. 92, 4261 (1990).

28. D. W. Norcross and M. J. Seaton, J. Phys. B6, 614 (1973).

29 J. A. Beswick and J. Jortner, J. Chem. Phys. 68, 2277 (1978); J. A. Beswick, G. Delgado-Barrio and J. Jortner, J. Chem. Phys. 70, 3895 (1979), J. A. Beswick and J. Jortner, Adv. Chem. Phys. 47 (part I), 363 (1981); G. E. Ewing, J. Chem. Phys. 71, 3143 (1979).

30. D. D. Evard, F. Thommen, J. I. Cline and K. C. Janda, J. Phys. Chem. 91, 2508 (1987); J. I. Cline, N. Sivakumar, D. D. Evard and K. C. Janda, J. Chem. Phys. 86, 1636 (1987); J. I. Cline, N. Sivakumar, D. D. Evard, C. R. Bieler, B. P. Reid, N. Halberstadt, S. R. Hair and J. C. Janda, J. Chem. Phys. 90, 2605 (1989).

31. J. C. Drobits, J. M. Skene and M. I. Lester, J. Chem. Phys. 84, 2896 (1986); J. M. Skene, J. C. Drobits and M. I. Lester, J. Chem. Phys. 85, 2329 (1986); J. C. Drobits and M. I. Lester, J. Chem. Phys. 88, 120 (1988); J. C. Drobits and M. I. Lester, J. Chem. Phys. 89, 4716 (1988).

32. G. Delgado-Barrio, P. Villarreal, P. Mareca and G. Albelda. J. Chem. Phys. 78, 280 (1983).

33. J. A. Beswick, G. Delgado-Barrio, P. Villarreal and P. Mareca, Faraday Discuss. Chem. Soc. 73, 406 (1982).

34. J. E. Kenny, K. E. Johnson. W. Sharfin and D. H. Levy, J. Chem. Phys. 72, 1109 (1980).

35. S. R. Hair, J. I. Cline, C. R. Bieler and K. C. Janda, J. Chem. Phys. 90, 2935 (1989). 